Ann. Sci. forest., 1973, 30 (4), 413-422.

\title{
INTÉRETT ET CONDITIONS D'APPORT DE TOURBE BLONDE EN PÉPINIÈRE AVANT REPIQUAGE D'ÉPICÉA COMMUN (PICEA ABIES) ET DE DOUGLAS (PSEUDOTSUGA MENZIESII)
}

\author{
Ph. LEROY et G. LÉVY \\ avec la collaboration technique de \\ G. Courrier et M. Guillemette
}

\begin{abstract}
Centre régional de la Propriété forestière, Lorraine-Alsace 57000 Metz - Le Ban St-Martin
Station de Recherches sur les Sols forestiers et la Fertilisation, Centre national de Recherches forestières, I.N.R.A., Champenoux 54370 Einville
\end{abstract}

\section{RÉSUMÉ}

Cette expérience a pour but de mettre en évidence l'intérêt et les meilleures conditions d'utilisation de tourbe blonde en pépinière. Les résultats concernent les deux premiers cycles de repiquage (de deux ans chacun).

Ils montrent qu'il a été inutile, et même parfois néfaste, pour les deux essences, d'effectuer des apports d'azote supérieurs aux exportations : la tourbe n'a pas bloqué l'azote. D'autre part, la tourbe a fourni des résultats au moins aussi bons que la terre de bruyère. Il sera cependant nécessaire de connaître l'arrière-effet de cette matière organique avant de la conseiller sans réserve aux pépiniéristes.

\section{INTRODUCTION}

La présence d'une certaine teneur (par exemple $5 \%$ ) de matière organique dans un sol de pépinière forestière peut présenter de nombreux avantages dans bien des cas; elle maintient la stabilité structurale du sol, libère progressivement certains éléments assimilables, en particulier l'azote, favorise la mycorrhization. C'est pourquoi, afin d'éviter que cette teneur ne diminue d'année en année par minéralisation, il est nécessaire d'effectuer périodiquement des apports de matière organique, par exemple au début de chaque cycle de semis ou de repiquage.

C'est une matière organique acide qu'il convient d'apporter dans une pépinière de résineux. Les tourbes blondes pourraient en constituer une source fort intéressante car d'approvisionnement facile, de maniement commode et de prix de revient relativement peu 
élevé. Mais, leur utilisation pose un problème important : leur rapport $\mathrm{C} / \mathrm{N}$ étant très élevé, l'azote destiné à la nutrition des semis risque d'être totalement bloqué par les microorganismes; de plus on ne peut guère compter sur une libération progressive d'azote.

Le but de l'expérience dont nous rendons compte ci-après est de déterminer la meilleure façon d'utiliser ces tourbes, dans une pépinière de repiquage, pour des épicéas communs (Picea abies) et des Douglas (Pseudotsuga menziesii). Les questions essentielles auxquelles nous nous efforcerons de répondre sont les suivantes :

- est-il nécessaire d'effectuer des apports d'azote destinés à compenser ce qui risque d'être fixé par la tourbe lors de son évolution éventuelle dans le sol (apports calculés dans l'hypothèse d'un abaissement du $\mathrm{C} / \mathrm{N}$ de la tourbe à 25); la nutrition azotée et la croissance pourraient alors s'effectuer normalement, d'autant plus que la tourbe serait transformée en un produit plus riche qui libérerait progressivement de l'azote.

- dans l'affirmative, quelle est la meilleure façon d'effectuer ces apports :

- sous forme d'engrais très soluble (ammonitrates) peu de temps avant le repiquage, en même temps que la tourbe?

Cette solution est la plus pratique et la moins onéreuse, mais cela risque d'occasionner des brûlures aux racines;

- sous forme d'engrais très soluble composté avec la tourbe plusieurs mois avant le traitement? L'azote est alors probablement intégré à des chaînes organiques et les brûlures ne sont plus à craindre.

- sous forme d'engrais lentement soluble, ce qui est une solution pratique et sans risque de brûlures, mais plus onéreuse que la première solution.

- quels sont les effets de la tourbe comparés à ceux de la terre de bruyère, autre matière organique utilisée en pépinière de résineux, mais dont le rapport $\mathrm{C} / \mathrm{N}$ est bien moins élevé, et que nous prendrons donc comme référence?

Le dispositif permettra également de répondre à certaines questions moins importantes posées par l'utilisation de la tourbe blonde.

Le critère d'efficacité des différents traitements choisis pour répondre à toutes ces questions sera la croissance en hauteur des plants complétée par l'analyse foliaire (test de nutrition).

Les résultats définitifs ne pourront être obtenus qu'après plusieurs cycles de repiquage, c'est-à-dire lorsque la tourbe aura constitué une forte proportion de l'humus du sol. Nous exposons dans ce mémoire les résultats des deux premiers cycles, principalement du second.

\section{I. - MATÉRIEL D’ÉTUDE ET PROTOCOLE EXPÉRIMENTAL}

1.1. - L'expérience se déroule dans 4 bâches de $30 \mathrm{~m}$ de long sur $1 \mathrm{~m}$ de large ( 2 bâches d'épicéas repiqués à 2 ans, 2 de Douglas repiqués à un an); le premier cycle a eu lieu en 1968-1969, le second en 1970-1971.

1.2. - Les bâches ont été remplies sur une hauteur de $40 \mathrm{~cm}$ avec un horizon A1 de sol brun acide sableux fin provenant de la parcelle 49 de la première série de la forêt domaniale de Bezangesla-Grande (54). C'est un mull acide $(5,1 \%$ de matière organique, $\mathrm{C} / \mathrm{N}$ de 17,3$)$. 


\section{3. - Les apports ont été effectués à l'aide des produits suivants :}

- terre de bruyère : provient de Sologne, contient environ $50 \%$ de matière organique, $\mathrm{pH}$ $=3,4, \mathrm{C} / \mathrm{N}$ voisin de 20 ;

- tourbe bonde : contient $99,4 \%$ de matière organique, $\mathrm{C} / \mathrm{N}=92$;

— ammonitrate à $33,5 \%$ de $\mathrm{N}$;

— nitroform : urée-formol à $38 \%$ de $\mathrm{N}$ soluble progressivement;

- superphosphate triple, sulfate de $\mathbf{K}$, carbonate de calcium, magnésie calcinée;

Les apports de matière organique ( $320 \mathrm{~kg}$ de terre de bruyère et $160 \mathrm{~kg}$ de tourbe sèches/ares) sont destinés théoriquement à compenser une minéralisation de l'humus du sol de $4 \%$ par an. En fait, malgré les conditions favorables (en particulier arrosages) il est presque certain que le taux de minéralisation réel est inférieur à cette valeur; mais il est intéressant, compte-tenu de l'objet de cette expérience, d'effectuer des apports élevés de tourbe; cela correspond d'ailleurs au cas d'un pépiniériste qui désirerait augmenter la teneur en matière organique de son sol.

Les apports d'azote ont deux objectifs : nutrition des plants et compensation de la fixation d'azote par la tourbe dans l'hypothèse où, au cours de son évolution, son $\mathrm{C} / \mathrm{N}$ s'abaisserait à 25 .

Les apports de $\mathrm{N}, \mathrm{P}, \mathrm{K}, \mathrm{Ca}, \mathrm{Mg}$ destinés à la nutrition des plants correspondent approximativement aux exportations de ces éléments effectuées après deux ans de repiquage $(\mathrm{N}: 2,4 \mathrm{~kg} / \mathrm{are}-$ $\left.\mathrm{P}_{2} \mathrm{O}_{5}: 0,70 \mathrm{~kg} / \mathrm{are}-\mathrm{K}_{2} \mathrm{O}: 1,04 \mathrm{~kg} / \mathrm{are}-\mathrm{CaO}: 1,92 \mathrm{~kg} / \mathrm{are}-\mathrm{MgO}: 0,24 \mathrm{~kg} / \mathrm{are}\right)$. N destiné à la nutrition des plants est apporté en 4 fois $\left(2\right.$ apports la $1^{\mathrm{re}}$ année $=1 / 3$ de la dose totale et 2 apports la seconde année $=2 / 3$ de la dose totale) lorsqu'il s'agit d'ammonitrates, en 2 fois (un apport par an, dont $1 / 3$ la $1^{\text {re }}$ année) pour le nitroform. $\mathrm{N}$ destiné à abaisser $\mathrm{C} / \mathrm{N}$ de la tourbe, ainsi que $\mathrm{P}, \mathrm{K}$, $\mathrm{Ca}, \mathrm{Mg}$ sont soit amenés dans les bâches peu de temps avant le repiquage (maximum un mois) en même temps que la tourbe mais indépendamment de celle-ci, soit compostés avec la tourbe plusieurs mois avant d'être mélangés au sol; ces composts sont arrosés et remués périodiquement. On effectue un arrosage copieux des bâches après ces apports.

1.4. - L'expérience principale, qui devrait permettre de répondre aux questions posées dans l'introduction, comporte 5 traitements numérotés $1,3,4,6,7$.

Deux traitements annexes ( 2 et 5 ) ont été ajoutés et seront commentés plus loin.

Chaque traitement comporte 4 répétitions (expérience en blocs), cela pour chacune des 2 essences.

Les traitements sont présentés au tableau 1.

Ce sont les résultats du $2^{\mathrm{e}}$ cycle qui devront surtout retenir notre attention : en effet, d'une part la quantité globale de tourbe mélangée au sol depuis le début de l'expérience est deux fois plus importante, d'autre part une erreur a été commise au cours de la réalisation du $1^{\text {er }}$ cycle : 1'azote destiné à la nutrition des plants n'a pas été apporté en $2^{\mathrm{e}}$ année aux traitements 1, 6 et 7 . Nous tenterons néanmoins d'interpréter les résultats de ce $1^{\text {er }}$ cycle.

\section{2. - RÉSULTATS}

La reprise est excellente pour tous les traitements; aucun d'entre eux n'a occasionné de mortalité significative aux plants.

Le tableau 2 présente les hauteurs moyennes des plants à la fin de chacun des deux cycles. Les hauteurs des semis au moment du repiquage étant à peu près identiques et faibles par rapport aux hauteurs finales, celles-ci représentent valablement la croissance pendant les 2 années de repiquage.

Les résultats des analyses foliaires sont reproduits au tableau 3.

\section{3. - COMMENTAIRES ET DISCUSSION}

Comme indiqué précédemment, nous commenterons principalement les résultats du $2^{\mathrm{e}}$ cycle. Rappelons que le critère retenu de comparaison des traitements est la croissance en hauteur. 
TABLEAU 1

\section{Les traitements}

The treatments

\begin{tabular}{|c|c|c|c|c|c|c|c|c|c|}
\hline \multirow{3}{*}{$\begin{array}{c}\text { Numéro } \\
\text { de } \\
\text { traitement }\end{array}$} & \multicolumn{2}{|c|}{ Matière organique } & \multicolumn{3}{|c|}{$\mathrm{N}$ destiné à abaisser $\mathrm{C} / \mathrm{N}$ de la tourbe } & \multicolumn{2}{|c|}{$\mathrm{P}, \mathrm{K}, \mathrm{Ca}, \mathrm{Mg}$} & \multicolumn{2}{|c|}{$\mathrm{N}$ destiné à la nutrition des plants } \\
\hline & \multirow{2}{*}{$\begin{array}{l}\text { Terre de } \\
\text { bruyère }\end{array}$} & \multirow{2}{*}{ Tourbe } & Composté & \multicolumn{2}{|c|}{ non composté } & \multirow{2}{*}{ Compostés } & \multirow{2}{*}{$\begin{array}{c}\text { Non } \\
\text { compostés }\end{array}$} & \multirow{2}{*}{ Ammonitrates } & \multirow{2}{*}{ Nitroform } \\
\hline & & & Ammonitrates & Ammonitrates & Nitroform & & & & \\
\hline 1 & $\mathrm{x}$ & & & & & & $\mathrm{x}$ & $\mathrm{x}$ & \\
\hline 2 & & & & & & & $\mathrm{x}$ & & $x$ \\
\hline 3 & & $\mathrm{x}$ & & & $\mathrm{x}$ & & $x$ & $\mathrm{x}$ & \\
\hline 4 & & $\mathrm{x}$ & $\mathrm{x}$ & & & & $\mathrm{x}$ & $\mathrm{x}$ & \\
\hline 5 & & $\mathrm{x}$ & $\mathrm{x}$ & & & $\mathrm{x}$ & & $\mathrm{x}$ & \\
\hline 6 & & $\mathrm{x}$ & & $\mathrm{x}$ & & & $\mathrm{x}$ & $\mathrm{x}$ & \\
\hline 7 & & $\mathrm{x}$ & & & & & $\mathrm{x}$ & $\mathrm{x}$ & \\
\hline
\end{tabular}




\section{TABLEAU 2}

Hauteur totale des plants deux ans après le repiquage $(\mathrm{cm})$ Total height of the plants two years after transplantation (in $\mathrm{cm}$ )

\begin{tabular}{|c|c|c|c|c|c|c|c|c|c|c|c|c|}
\hline & \multicolumn{7}{|c|}{ Numéro du Traitement } & \multicolumn{2}{|c|}{ F théorique } & \multirow[t]{2}{*}{ F calculé } & \multicolumn{2}{|c|}{ Différences significatives } \\
\hline & 1 & 2 & 3 & 4 & 5 & 6 & 7 & à $5 \%$ & à $1 \%$ & & à $5 \%$ & à $1 \%$ \\
\hline Épicéa $1^{\mathrm{er}}$ cycle & 28,4 & 29,7 & 30,7 & 28,5 & 29,3 & 28,3 & 30,0 & 2,66 & 4,01 & 0,29 & & \\
\hline Douglas $1^{\mathrm{er}}$ Cycle & 63,0 & 70,5 & 72,1 & 69,3 & 61,9 & 69,3 & 68,4 & 4,28 & 8,47 & 2,38 & & \\
\hline Épicéa $2^{\mathrm{e}}$ Cycle & 35,1 & 33,8 & 32,0 & 33,6 & 29,4 & 34,1 & 37,9 & 2,66 & 4,01 & $3,38^{*}$ & $\begin{array}{l}7>4 \\
5<1,6,2\end{array}$ & $7>5$ et 3 \\
\hline Douglas $2 \mathrm{e}$ Cycle & 88,2 & 89,9 & 87,1 & 89,5 & 89,6 & 89,4 & 95,0 & 2,66 & 4,01 & 1,03 & & \\
\hline
\end{tabular}


TABLEAU 3

Analyses foliaires à la fin de chaque cycle

Foliar analysis at the end of the two cycles

\begin{tabular}{|c|c|c|c|c|c|c|c|c|c|c|c|c|c|c|}
\hline \multirow[b]{2}{*}{ Traitement } & \multicolumn{7}{|c|}{ Épicéa $1^{\mathrm{er}}$ cycle } & \multicolumn{7}{|c|}{ Douglas $1^{\text {er }}$ Cycle } \\
\hline & 1 & 2 & 3 & 4 & 5 & 6 & 7 & 1 & 2 & 3 & 4 & 5 & 6 & 7 \\
\hline N (\%) & 1,88 & 1,98 & 2,09 & 1,99 & 1,55 & 1,94 & 1,97 & 1,62 & 1,69 & 1,58 & 1,48 & 1,36 & 1,51 & 1,51 \\
\hline P (\%) & 0,26 & 0,29 & 0,29 & 0,29 & 0,28 & 0,30 & 0,30 & 0,27 & 0,23 & 0,27 & 0,27 & 0,29 & 0,24 & 0,27 \\
\hline $\mathrm{K}(\%)$ & 0,69 & 0,59 & 0,59 & 0,68 & 0,66 & 0,61 & 0,66 & 0,93 & 0,93 & 0,95 & 0,97 & 0,98 & 0,93 & 0,98 \\
\hline $\mathrm{Ca}(\%)$ & 1,06 & 1,14 & 1,02 & 0,98 & 0,94 & 1,09 & 1,02 & 0,43 & 0,40 & 0,40 & 0,45 & 0,40 & 0,40 & 0,45 \\
\hline $\mathrm{Mg}(\%)$ & 0,06 & 0,08 & 0,09 & 0,06 & 0,05 & 0,08 & 0,07 & 0,08 & 0,06 & 0,06 & 0,08 & 0,09 & 0,09 & 0,09 \\
\hline \multirow[t]{2}{*}{$\mathrm{Mn}(\% / 00)$} & 2,43 & 1,85 & 1,92 & 1,30 & 2,05 & 1,79 & 1,41 & 1,49 & 0,75 & 1,55 & 1,06 & 1,44 & 1,98 & 1,86 \\
\hline & \multicolumn{7}{|c|}{ Épicéa $2^{e}$ Cycle } & \multicolumn{7}{|c|}{ Douglas $2^{\mathrm{e}}$ Cycle } \\
\hline Traitement & 1 & 2 & 3 & 4 & 5 & 6 & 7 & 1 & 2 & 3 & 4 & 5 & 6 & 7 \\
\hline $\mathrm{N}(\%)$ & 1,60 & 1,60 & 1,80 & 1,90 & 1,79 & 1,81 & 1,73 & 1,67 & 1,58 & 1,77 & 1,70 & 1,55 & 1,58 & 1,81 \\
\hline $\mathrm{P}(\%)$ & 0,17 & 0,18 & 0,17 & 0,18 & 0,17 & 0,18 & 0,19 & 0,13 & 0,14 & 0,14 & 0,13 & 0,13 & 0,13 & 0,13 \\
\hline $\mathrm{K}(\%)$ & 0,40 & 0,43 & 0,38 & 0,40 & 0,40 & 0,39 & 0,40 & 0,59 & 0,66 & 0,57 & 0,58 & 0,56 & 0,56 & 0,61 \\
\hline $\mathrm{Ca}(\%)$ & 0,89 & 0,97 & 0,92 & 0,95 & 0,90 & 0,96 & 0,90 & 0,42 & 0,40 & 0,45 & 0,44 & 0,40 & 0,42 & 0,45 \\
\hline $\operatorname{Mg}(\%)$ & 0,06 & 0,08 & 0,06 & 0,07 & 0,06 & 0,07 & 0,08 & 0,10 & 0,10 & 0,11 & 0,11 & 0,10 & 0,11 & 0,11 \\
\hline $\operatorname{Mn}(\%)$ & 1,11 & 1,11 & 1,36 & 1,39 & 1,44 & 1,47 & 1,06 & 0,38 & 0,43 & 1,11 & 0,54 & 0,50 & 0,80 & 0,48 \\
\hline
\end{tabular}




\section{1. - L'Épicéa}

\subsection{Le $2^{\mathrm{e}}$ cycle.}

- Le traitement 7 est significativement supérieur aux traitements 3 et 4; il est assez nettement supérieur, bien que non significativement, au traitement $6:$ il a donc été inutile, et dans la plupart des cas même néfaste, d'essayer d'abaisser le $\mathrm{C} / \mathrm{N}$ de la tourbe, quelle que soit la méthode retenue. Les résultats fournis par ces trois méthodes (traitements $3-4$ - 6) sont à peu près identiques; l'apport d'une forte quantité d'ammonitrates au sol peu avant le repiquage n'a donc pas occasionné de dommages aux plants (par brûlures des racines) par rapport aux autres modes d'apport d'une quantité identique d'azote; il est vrai que des arrosages ont été effectués après cet apport d'ammonitrates; le nitroform ne s'est pas avéré préférable, pour cet objectif, aux ammonitrates.

Les traitements comportant un apport d'engrais destinés à abaisser le $\mathrm{C} / \mathrm{N}$ de la tourbe présentent la teneur foliaire en azote la plus élevée, ce qui est logique; celles des autres traitements, en particulier 7, paraissent pourtant convenables. Cet apport élevé crée peutêtre un déséquilibre alimentaire au détriment du phosphore en particulier.

- La comparaison des traitements 1 et 7 montre qu'un apport de tourbe ne s'est pas révélé néfaste par rapport à un apport de terre de bruyère (presque au contraire). Ce résultat est très intéressant pour les raisons pratiques mentionnées précédemment.

La teneur des feuilles en azote confirme d'ailleurs parfaitement le résultat précédent.

\subsection{Le I $^{\mathrm{er}}$ cycle.}

Malgré le fait que l'apport de $\mathrm{N}$ destiné à la nutrition des plants n'ait pas été réalisé au cours de la $2^{\mathrm{e}}$ année pour certains traitements, les résultats permettent de confirmer les conclusions essentielles du $2^{\mathrm{e}}$ cycle.

\section{2. - Le Douglas}

\subsection{Le $2^{\mathrm{e}}$ cycle.}

Le test $\mathrm{F}$ ne donne pas de résultat significatif. En fait, si les différences entre les moyennes des traitements autres que le $7^{\mathrm{e}}$ sont faibles, ces moyennes sont toutes assez nettement inférieures à celles du 7 e traitement : comme pour l'Épicéa, un apport d'azote destiné à abaisser $\mathrm{C} / \mathrm{N}$ de la tourbe s'est avéré pour le moins inutile.

Comme pour l'Épicéa également, un important apport d'engrais azotés solubles peu avant le repiquage n'a pas occasionné de dommages aux plants comparé à l'apport d'une quantité identique d'azote effectué d'une manière théoriquement moins dangereuse.

Les plants du traitement 7 présentent une teneur foliaire en azote au moins équivalente à celle des traitements 3,4 et $6:$ comme dans le cas de l'Épicéa, il n'y a pas eu de blocage d'azote par la tourbe. 
L'apport de matière organique sous forme de tourbe ne s'est pas révélé plus défavorable au contraire, qu'un apport sous forme de terre de bruyère; la nutrition azotée des plants semble même plus favorable dans le premier cas.

Les résultats essentiels du $2^{\mathrm{e}}$ cycle sont donc équivalents à ceux de l'Épicéa.

\subsection{Le $1^{\text {er }}$ cycle.}

Les résultats essentiels confirment, comme pour l'Épicéa, ceux du $2^{\mathrm{e}}$ cycle.

\section{3. - Remarques générales quant au comportement de la tourbe}

L'examen de la nutrition azotée et de la croissance en hauteur, pour les deux essences, laisse supposer que la tourbe s'est comportée en substrat " inerte » : il n'y a pas eu blocage de l'azote. Il semble même qu'au moins dans certains cas la tourbe ait eu de ce point de vue un comportement plus intéressant que la terre de bruyère : celle-ci a peut-être bloqué une partie de l'azote destiné à la nutrition des plants.

Les résultats comparatifs fournis par tourbe et terre de bruyère se modifieront peut-être au cours des cycles suivants. On peut supposer sous toutes réserves, à long terme, un avantage et un inconvénient de la première par rapport à la seconde; l'avantage : son taux de minéralisation est sans doute très faible, done très peu d'apports nouveaux seront nécessaires après un certain nombre de cycles; en contre-partie, la libération progressive d'azote, au cours de la saison de végétation, devrait être nettement plus importante à partir d'un humus formé par apport de terre de bruyère.

\section{4. - Les traitements annexes}

Le traitement 2, qui comporte une dose d'azote destinée à la nutrition des plants supérieure à celle des autres traitements pour compenser l'apport d'azote libéré dans ceux-ci par minéralisation de la matière organique, a surtout un objectif à long terme : il s'agit de se rendre compte si l'emploi d'un engrais azoté lentement soluble permet d'éviter des apports périodiques de matière organique.

Les résultats du $2^{\text {e }}$ cycle ne présentent donc encore qu'assez peu d'intérêt car susceptibles de varier considérablement à l'avenir, au fur et à mesure de la minéralisation de l'humus de départ. Remarquons seulement que, pour les deux essences, un seul apport annuel de nitroform s'est révélé aussi efficace qu'un enfouissement de terre de bruyère suivi de deux apports annuels d'ammonitrates. Cela ne semble plus le cas si la tourbe remplace la terre de bruyère.

Dans le traitement 5, on composte avec la tourbe non seulement l'azote destiné à abaisser $\mathrm{C} / \mathrm{N}$ de celle-ci, comme dans le traitement 4 , mais aussi les engrais à $\mathrm{P}, \mathrm{K}, \mathrm{Ca}$ et $\mathrm{Mg}$. Ce procédé présenterait l'avantage de simplifier les apports entre l'arrachage des plants et les nouveaux repiquages.

En fait, les résultats montrent que ce compostage est aussi inutile ou même néfaste, et parfois même plus, que les autres traitements destinés à abaisser le rapport $\mathrm{C} / \mathrm{N}$ de la tourbe. 


\section{4. - CONCLUSIONS}

Les résultats essentiels concordent, à la fois pour les deux essences et les deux cycles. On a pu sans danger immédiat effectuer des apports de tourbe blonde en pépinière avant des repiquages d'Épicéa et de Douglas; des adjonctions d'azote, que l'on a effectuées en raison du $\mathrm{C} / \mathrm{N}$ élevé de la tourbe, se sont avérées inutiles.

Néanmoins, avant de conseiller sans réserve l'utilisation de tourbe blonde aux pépiniéristes, il serait nécessaire de connaître l'arrière effet éventuel de cette matière organique; aussi cette expérience sera-t-elle encore suivie pendant plusieurs cycles.

Reçu pour publication en novembre 1973.

\section{SUMMARY}

INTEREST AND CONDITIONS OF ADDITION OF SPHAGNUM

PEAT IN NURSERY BEFORE TRANSPLANTING NORWAY SPRUCE

AND DOUGLAS-FIR

The purpose of this experiment is to point out the interest and the best way of using Sphagnum peat in nursery. This addition is supposed to compensate the progressive mineralisation of soil organic matter.

All treatments include mineral additions corresponding to the nutrients contained in the plants when they are rooted out. Some treatments include supplementary additions of nitrogen realized different ways; the purpose of these additions is to compensate the very hight $\mathrm{C} / \mathrm{N}$ ratio of the peat. Nitrogen is brought either as $\mathrm{NO}_{3} \mathrm{NH}_{4}$, either as nitroform (a slowly soluble fertilizer).

The treatments with peat are compared with a treatment includind heather soil, which had previously given satisfactory results.

The results exposed in this paper concern the first two transplanting cycles (each cycle is two years long). They show that it is useless, and even sometimes harmful, for the two species, to bring nitrogen in quantity higher than what is absorbed by the plants : peat has not retained nitrogen. On the other hand, peat gives at least as good results as heather soil.

\section{ZUSAMMENFASSUNG}

BEDEUTUNG UND EINBRINGUNGSMÖGLICHKEITEN VON TORFMULL IM PFLANZGARTEN VOR DER VERSCHULUNG VON FICHTE UND DOUGLASIE

Die vorliegende Untersuchung behandelt die Bedeutung und die optimalen Verwendungsmöglichkeiten von Torfmull im Pflanzgarten. Die Ergebnisse beziehen sich auf die zwei ersten Zweijahresperioden nach der Verschulung.

Es zeigte sich, dass eine Stickstoffdüngung die den Stickstoffersatz übersteigt nicht notwendig ist und manchmal sogar schädigend wirkt und dass der Torfmull den Stickstoff nicht festlegt. Die Einbringung von Torfmull ergab zumindest ebenso gute Resultate als die Verwendung von Torferde. 


\section{RÉFÉRENCES BIBLIOGRAPHIQUES}

BenZian B., 1965. - Experiments on nutrition problems in forest nurseries. 2 vol. Buil. Forestry Comm., London, 37, $251+265$.

KaUnisto S., 1968. - (Fertilized garden peat as a substratum for germinating forest tree seeds). Suo, Helsinki, 19 (3/4), 57-69 (in For. Abstr, 1969, 30 (4) 5582).

Leroy Ph., Lévy G. et Strullu D. G., 1973. - Action de la matière organique et des engrais minéraux en pépinière sur la croissance et les caractères foliaires du Douglas. Annales Sci. forest., sous presse.

Muller J., 1971. - Effets des amendements organiques, tourbes, écorces de résineux et de feuillus, sur l'évolution de l'azote minéral. C. R. Séances Acad. Agric. Fr., 13, 1123-1133.

STrullu D. G., 1972. - Recherches sur le comportement du Douglas en pépinière (Pseudotsuga menziesii (Mirb) Franco). (Thèse $3^{\mathrm{e}}$ cycle 1970) - Botanica Rhedonica, 12, 108. 